\title{
Detection of circular RNA expression and related quantitative trait loci in the human dorsolateral prefrontal cortex
}

\author{
Zelin Liu' ${ }^{1}$, Yuan Ran², Changyu $\mathrm{TaO}^{3} \mathbb{B}$, Sichen $\mathrm{Li}^{4}$, Jian $\mathrm{Chen}^{2}$ and Ence Yang ${ }^{1,3,5^{*}}$ (D)
}

\begin{abstract}
Background: Circular RNAs (circRNAs) are implicated in various biological processes. As a layer of the gene regulatory network, circRNA expression is also an intermediate phenotype bridging genetic variation and phenotypic changes. Thus, analyzing circRNA expression variation will shed light on molecular fundamentals of complex traits and diseases.

Results: We systematically characterize 10,559 high-quality circRNAs in 589 human dorsolateral prefrontal cortex samples. We identify biological and technical factors contributing to expression heterogeneity associated with the expression levels of many circRNAs, including the well-known circRNA CDR1as. Combining the expression levels of circRNAs with genetic cis-acting SNPs, we detect 196,255 circRNA quantitative trait loci (circQTLs). By characterizing circQTL SNPs, we find that partial circQTL SNPs might influence circRNA formation by altering the canonical splicing site or the reverse complementary sequence match. Additionally, we find that a subset of these circQTL SNPs is highly linked to genome-wide association study signals of complex diseases, especially schizophrenia, inflammatory bowel disease, and type II diabetes mellitus.
\end{abstract}

Conclusions: Our results reveal technical, biological, and genetic factors affecting circRNA expression variation among individuals, which lead to further understanding of circRNA regulation and thus of the genetic architecture of complex traits or diseases.

Keywords: Circular RNA, circQTLs, Expression variation, GWAS

\section{Background}

Circular RNAs (circRNAs) are a class of recently identified long noncoding RNAs with a covalently closed continuous loop structure formed via back-splicing that have neither a 5' cap nor a 3' polyadenylated tail [1]. Thousands of circRNAs have been identified in humans and other species [2]. A handful of circRNAs have been functionally elucidated, and these functions include acting as sponges for microRNAs [3, 4], competing for RNA-binding protein [5], and even translating into protein [6,7]. circRNAs are thought to play crucial roles in multiple cellular processes

\footnotetext{
* Correspondence: yangence@pku.edu.cn

'Institute of Systems Biomedicine, Department of Medical Bioinformatics, School of Basic Medical Sciences, Peking University Health Science Center, Beijing 100191, People's Republic of China

${ }^{3}$ Department of Microbiology and Infectious Disease Center, School of Basic Medical Sciences, Peking University Health Science Center, Beijing 100191, People's Republic of China

Full list of author information is available at the end of the article
}

and disease pathogenesis based on their tissue- and development-specific manner of expression $[3,4,8]$. As a layer of the gene regulatory network, circRNA expression is also an intermediate phenotype bridging genetic variants and phenotypic changes. Thus, understanding circRNA expression variation will shed light on the molecular fundamentals of complex traits and diseases.

However, due to the intrinsic circRNA characteristic of lacking a polyadenylated tail, circRNA expression has been underestimated by RNA-seq using the polyadenylated selection method. Several studies have identified circRNAs and quantified their abundance at the transcript level $[9,10]$, but small sample sizes limited their efforts to systemically dissect changes in circRNA expression. Data from the CommonMind Consortium (CMC) provide an opportunity to decipher circRNA expression variation. The CMC comprises nearly 600 brain samples obtained from the autopsies of individuals 
with and without severe psychiatric disorders [11]. Data generated from the CMC include SNP genotypes and gene expression (RNA-seq) as well as other functional genomic data. More importantly, CMC RNA sequencing libraries were prepared by ribosomal RNA depletion, which facilitated the analysis of circRNA expression.

In the current study, we utilized genotype and dorsolateral prefrontal cortex (DLPFC) expression data provided by the CMC to exploit circRNA expression variation arising from genetic, biological, and technical factors. We detected more than 10,000 high-quality circRNAs in the samples using stringent methodologies. We then highlighted the effects of covariates on circRNA expression variation. By circQTL analysis, we characterized the cis-regulation of circRNA and found that circQTL SNPs were enriched among disease risk loci. Our findings will further our understanding of the genetic architectures of complex traits or diseases.

\section{Results}

\section{Identification of circRNAs in the human brain}

We systemically identified circRNAs by analyzing Ribo-Zero RNA-seq data from postmortem DLPFC samples collected from 258 schizophrenia (SCZ) patients, 54 affective/mood disorder (AFF) patients, and 277 controls (Table 1) downloaded from the CMC database [11]. We first de novo identified circRNAs originating from canonical GT-AG splicing using CIRI [12, 13] (Fig. 1a), which achieved better balanced performance between precision and sensitivity [14] and reported both circular junction counts and the circular ratio (Fig. 1b). By using a loose criterion (circular junction counts $\geq 1$ ), we detected 9776 to 63,781 (median 31,286) potential back-splicing sites and 30,188 to 577,672 (median 143,573) potential circular junction counts (Fig. 1c) for each sample. These junction counts account for average about $0.8 \%$ of the total gene counts,

Table 1 Sample description

\begin{tabular}{|c|c|c|c|}
\hline Variables & Controls & SCZ & AFF \\
\hline Subjects & 277 & 258 & 54 \\
\hline Male sex, N (\%) & $158(57.0)$ & $164(63.6)$ & $27(50.0)$ \\
\hline 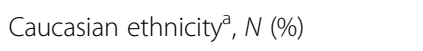 & $205(74.0)$ & $209(81.0)$ & $51(94.4)$ \\
\hline Age of death ${ }^{\mathrm{b}}$ (years), mean (SD) & $65.1(18.9)$ & $68.7(16.5)$ & $51.5(16.1)$ \\
\hline Postmortem interval (h), mean (SD) & $13.6(7.9)$ & $20.8(13.5)$ & $19.3(7.1)$ \\
\hline RNA integrity number, mean (SD) & $7.8(0.8)$ & $7.4(0.9)$ & $7.9(0.7)$ \\
\hline Gene aligned reads $\left(10^{6}\right)$, mean (SD) & $20.1(6.5)$ & $17.7(5.7)$ & $21.0(4.5)$ \\
\hline $\begin{array}{l}\text { Linear junction aligned reads }\left(10^{5}\right) \text {, } \\
\text { mean (SD) }\end{array}$ & $57.2(32.6)$ & $47.7(26.7)$ & $56.4(24.0)$ \\
\hline $\begin{array}{l}\text { Circular junction aligned reads }\left(10^{4}\right) \text {, } \\
\text { mean (SD) }\end{array}$ & $16.9(7.3)$ & $15.0(6.7)$ & $15.3(5.9)$ \\
\hline
\end{tabular}

${ }^{\mathrm{a}}$ Genetic inferred Caucasian ethnicity

${ }^{b}$ Age of death $\geq 90$ was considered 90 due to the limited information in the original data source which are higher than most of other tissues and cell lines (Additional file 1: Table S1; Additional file 2: Figure S1). Then, we screened out circRNAs expressed in the human brain (circular junction counts $\geq 1$ in more than half of individuals) with a potential function instead of byproducts of linear splicing (average circular ratio $\geq 0.05$ ). Finally, we identified 10,559 high-quality circRNAs (Additional file 3: Table S2), half of which were detected in 495 samples at least (Fig. 1d), and 781 (7.4\%) of which expressed higher than their linear counterparts (circRNA ratio $>0.5$ ). About $99.7 \%(10,526$ of 10,559$)$ predicted high-quality circRNAs could also be detected by find_circ [4] and/or CIRCexplorer [15] (Additional file 3: Table S2). Approximately 11.6\% (1229 of 10,559) of the high-quality circRNAs were not mentioned in previous studies $[2,9]$. Among them, 13 highly expressed circRNAs were detected in two human glioma stem cell lines (GSC11 and G118, details in the "Materials and methods" section) [16]. And 11 of 13 (85\%) back-splicing events were experimentally validated by two rounds RT-PCR with divergent primers (Fig. 1e; Additional file 4: Table S3). Then, we further confirmed our analysis with five randomly selected products by Sanger sequencing (Fig. 1f; Additional file 2: Figure S2). Together, these results supported the existence of 1229 novel circRNAs in CMC dataset.

Consistent with a previous study on circRNAs in HEK293 cells [4], 87.1\% of the circRNAs (9198 of 10,559) were located in exonic regions, followed by intronic (7.0\%) and intergenic $(5.9 \%)$ regions in the human brain. Most (96.7\%) exonic circRNAs overlapped with the coding sequence region (Fig. 1g) according to the GENCODE annotation. The median number of exons and median length of exonic circRNAs were determined to be five and $780 \mathrm{bp}$, respectively (Additional file 2: Figure S3).

The 9935 exonic and intronic circRNAs were derived from 3916 genes, $45.9 \%$ of which generated only one circRNA (Additional file 2: Figure S4). To analyze the functions of circRNA host genes, the 3916 host genes were uploaded to DAVID tools [17] (https://david.ncifcrf.gov/) as a test gene list, and 16,423 expressed genes from the same CMC sample set [11] served as the background. Alternative splicing (Benjamini-corrected $P=4.99 \times 10^{-78}$, Fisher's exact test (FET)) and splice variants (Benjamini-corrected $P=3.65 \times 10^{-65}$, FET) were the most significantly enriched among the host genes (Additional file 5 : Table S4), consistent with the existing knowledge that back-splicing is correlated with linear splicing [18]. A previous study observed that circRNAs are enriched in synapses in the mouse brain [19]. Interestingly, postsynaptic membrane (Benjamini-corrected $P=0.0083, \mathrm{FET}$ ) and postsynaptic density (Benjamini-corrected $P=0.039$, FET) were enriched in our detected host genes, which also suggests that circRNAs play important roles in synaptic function. 
A

\begin{tabular}{|c|c|c|c|c|}
\hline \multirow{2}{*}{$\begin{array}{l}589 \text { CMC Ribo-Zero } \\
\text { RNA-seq data }\end{array}$} & \multirow{2}{*}{ BWA mapping } & \multirow{2}{*}{$\begin{array}{c}\text { CIRI detection \& } \\
\text { quantification }\end{array}$} & average circRNA ratio $\geq 5 \%$ & \multirow{2}{*}{$\begin{array}{c}10,559 \text { high-quality } \\
\text { circRNAs }\end{array}$} \\
\hline & & & circular junction & \\
\hline
\end{tabular}

B
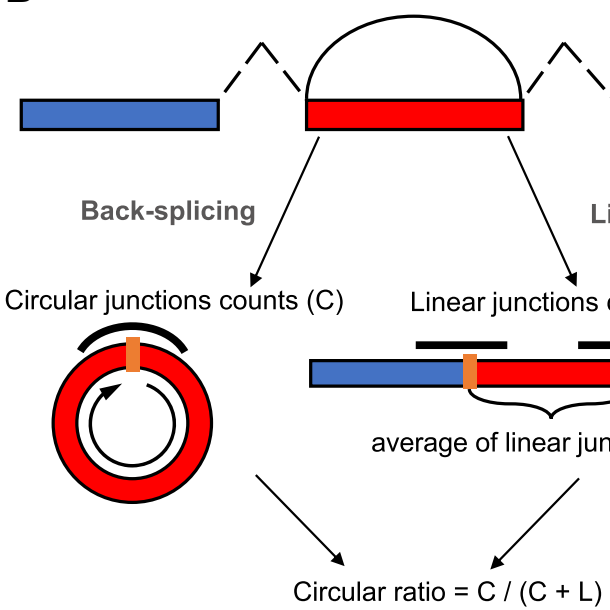

D

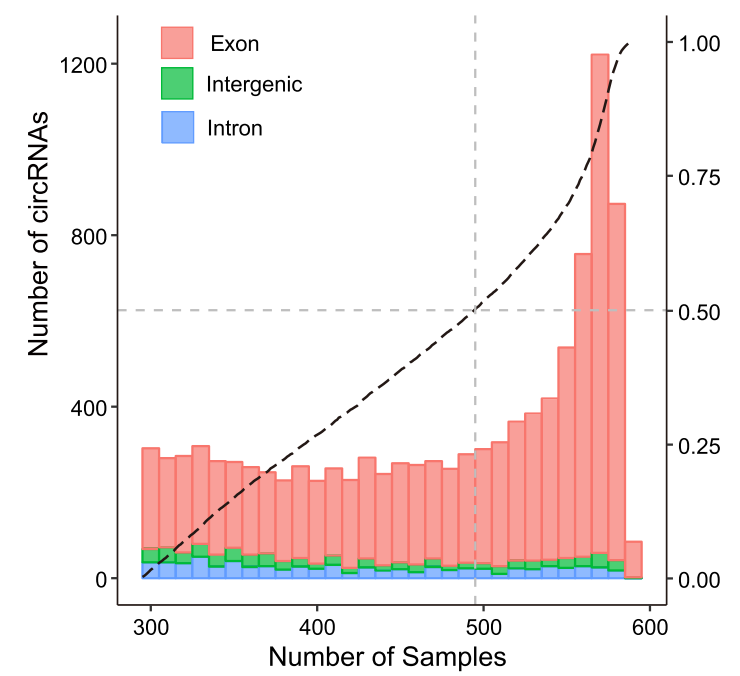

$\mathbf{F}$

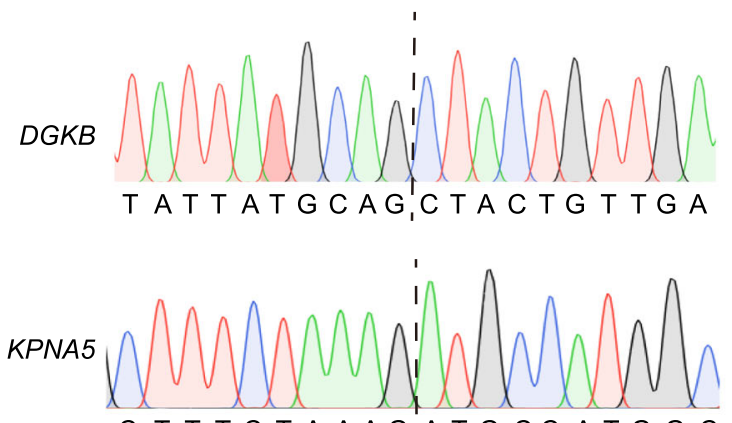

C T T T C TAAAG,ATG C C ATG G C
C

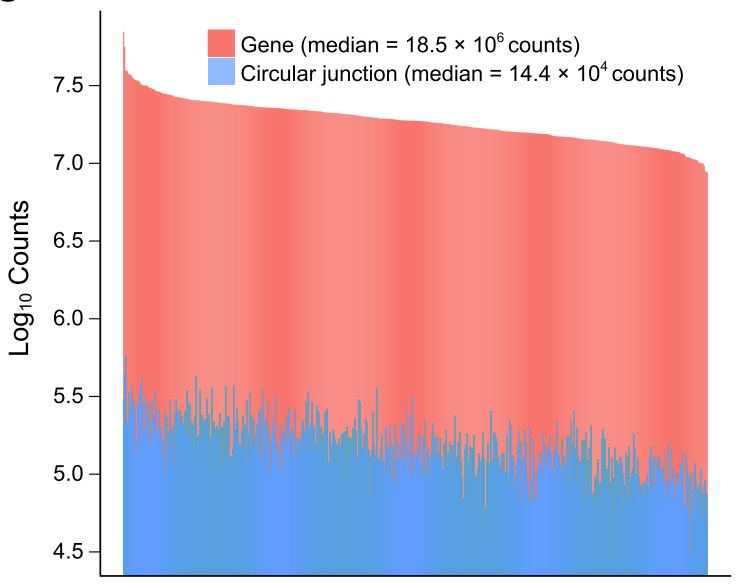

Samples sorted by gene depth

E

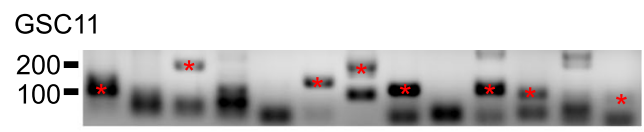

G118

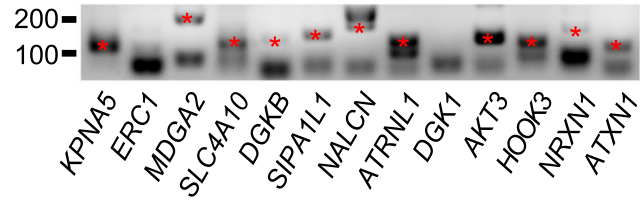

G

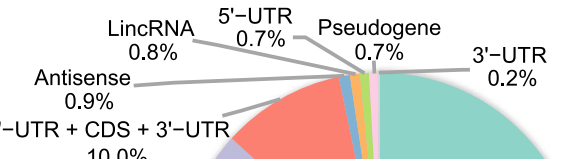

$10.0 \%$

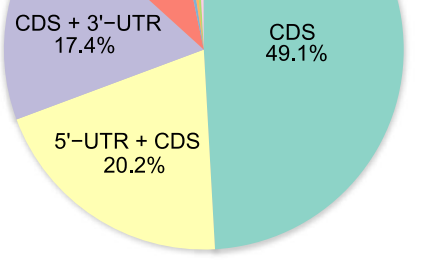

Fig. 1 (See legend on next page.) 
(See figure on previous page.)

Fig. 1 Profiling of circRNAs in the DLPFCs of 589 individuals. a The analysis pipeline to identify and quantify high-quality circRNAs. b A schematic diagram for calculating the circular ratio. c The stack diagram showing distributions of total gene counts and potential circular junction counts. The counts are log base 10 transformed on the $y$-axis. $\mathbf{d}$ The sample distribution for existing circRNAs in exonic, intronic, and intergenic regions. The black dashed line indicates the cumulative data fraction. e Gel analysis shows the target RT-PCR product bands. Red star points indicate the target band. f Two Sanger sequencing examples showed that RT-PCR products spanned the circular junction. Dashed line indicates the circular junction site. $\mathbf{g}$ Annotations of genomic regions mapping to inferred exonic circRNAs. CDS: coding sequence; lincRNA: long intergenic noncoding RNA; UTR: untranslated region

\section{Normalization of circRNA expression and evaluation of covariate effects}

Considering the stability differences between circular and linear RNAs, we measured the circRNA expression levels with voom [20] by circular junction counts per million circular junction reads (circCPM), which normalized the sequencing depths among samples (Fig. 2a). Similar to a previous study [11], both the technical (institution, library preparation batch (LIB), postmortem interval (PMI), RNA integrity number (RIN)) and the biological (age of death (AOD), ethnicity, gender, SCZ) effects were considered as covariates. We applied the linear regression utilities in the

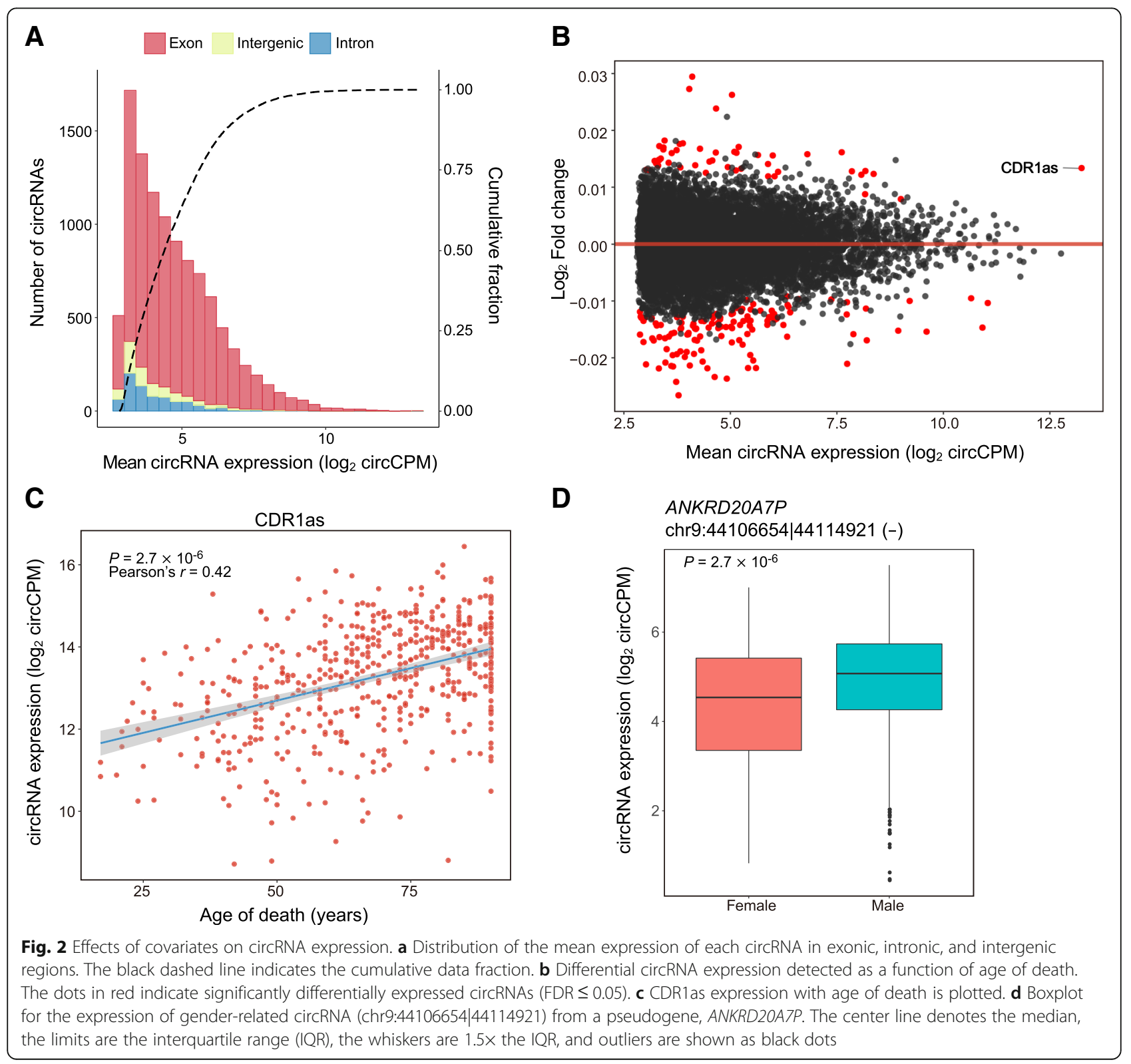


limma [21] package to evaluate the effects of these factors on each circRNA. In contrast, we used the same method to analyze the effects of covariates on gene expression normalized by gene counts per million gene reads (gCPM; see the "Materials and methods" section). Compared to linear RNAs, the expression of circRNAs is less affected by all the covariates than linear RNAs (Table 2).

The library batch is the most significant factor impacting both circRNA expression and gene expression. Compared to random sampling, LIB-associated circRNAs have higher expression levels $\left(P<1 \times 10^{-4}\right.$, bootstrapping) and shorter median lengths $\left(P<1 \times 10^{-4}\right.$, bootstrapping; Additional file 2: Figure S5), suggesting that the length of a circRNA may be related to circRNA stabilization. A tiny fraction of circRNAs was expressed in association with institution (2.8\%), PMI (0.02\%), and RIN (0.27\%), consistent with circRNAs being more stable than linear RNAs.

For age of death, 49 circRNAs exhibited increased expression and 126 circRNAs showed decreased expression with age (Fig. 2b; Additional file 6: Table S5). Among 123 circRNAs originating from an explicit host gene, 32 are AOD-related, while their host genes are not, suggesting that the roles of these circRNAs in aging are independent of their counterparts. Interestingly, a famous brain-enriched circRNA, CDR1as, which is known as a sponge for miR-7 regulating various diseases, such as cancer [22] and neurodegenerative diseases [23], showed increased expression with age (Fig. 2c), implying that CDR1as may be involved in aging of the brain.

For gender, 21 of 35 differential circRNAs were located on sex chromosomes. After removing circRNAs from the sex chromosomes and readjusting the $P$ values of the remaining circRNAs, we found only one circRNA that was differentially expressed (DE). This circRNA was highly expressed in males and originated from the pseudogene ANKRD20A7P (Fig. 2d), which is especially highly expressed in testes [24].

For schizophrenia, we did not detect any DE circRNAs after false discovery rate (FDR) correction (top FDR $=0.53$, $P=1.1 \times 10^{-4}$ ) between cases and controls, or randomly selected samples (100 vs. 100, 50 vs. 50) for 1000 times (see details in the "Materials and methods" section). Although gender differences exist in susceptibility of SCZ [25], neither male-specific nor female-specific DE circRNAs were detected. In the previous analysis, we filtered out circRNAs in a sample set including both SCZ and control populations. To further mine disease-specific expressed
circRNAs, we reanalyzed 303 circRNAs that were expressed in either SCZ or control populations. With the same procedure, we still failed to detect any DE circRNAs (see details in the "Materials and methods" section). Considering that there are hundreds of $\mathrm{DE}$ genes but no $\mathrm{DE}$ circRNAs, we compared the distributions of effect size between genes and circRNAs and found that the effect size of circRNAs is smaller than genes (Additional file 2: Figure S6). Thus, it requires 275 cases vs. 275 controls to detect $\mathrm{DE}$ genes, but 435 cases $v s .435$ controls to detect DE circRNAs, at the significance level of $80 \%$ power (Bonferroni-corrected $P<0.05$, Student's $t$ test; see details in the "Materials and methods" section). As there are only 258 SCZ and 277 control samples in CMC dataset, a larger sample size or more sensitive statistical methods may be required to detect DE circRNAs between SCZ and control.

\section{Effects of genetic variation on circRNA expression}

To measure how circRNA expression is regulated by genetic variations, we analyzed the association of covariateadjusted circRNA expression with genetic variants, i.e., circRNA quantitative trait loci (circQTLs). Similar to cis-sQTLs for alternative splicing [26, 27], we selected circRNAs in combination with high-quality (imputation quality score $\geq 0.8$ ) and common SNPs (minor allele frequency $(\mathrm{MAF}) \geq 0.05)$ in the $\pm 100-\mathrm{kb}$ region of back-splicing sites to identify cis-circQTLs by using Matrix eQTL [28]. After permutation and $q$ value [29] correction to control the FDR, we identified 251,374 circQTLs associated with 2790 circRNAs.

A weak correlation has been reported between circRNAs and linear RNA expression [30, 31]. To exclude circQTL SNPs that regulate host gene expression rather than circRNA expression, we further refined the circQTL SNPs regulating exonic and intronic circRNAs by reanalyzing cis-circQTLs with a circular ratio instead of circCPM. Following the same pipeline, we detected 228,578 circQTLs, 166,975 (73.05\%) of which were identified in both (Fig. 3a, b) and with concordant effects (Fig. 3c). In addition to the intergenic circQTLs, which lack host genes, we finally identified a total of 196,255 circQTLs associated with 2086 circRNAs involving 1269 genes (Additional file 7: Table S6).

To estimate the proportion of significant circQTLs that are also eQTLs, we selected the most significant circQTL SNP for each circRNA (max-circQTL) to characterize circQTLs. Compared with eQTLs identified

Table 2 The numbers of circRNAs and genes affected by technical and biological factors at an FDR $<0.05$

\begin{tabular}{|c|c|c|c|c|c|c|c|c|}
\hline Type & Institution & LIB & PMI & RIN & AOD & Ethnicity & Gender & SCZ \\
\hline $\operatorname{circRNA}(N=10,559)$ & $300(2.8 \%)$ & 4143 (39\%) & $2(0.02 \%)$ & $28(0.27 \%)$ & $175(1.7 \%)$ & $249(2.4 \%)$ & $35(0.33 \%)$ & $0(0 \%)$ \\
\hline Gene $(N=16,422)$ & 13,291 (81\%) & $14,759(90 \%)$ & $4033(24 \%)$ & $11,121(68 \%)$ & $9668(59 \%)$ & $1097(6.7 \%)$ & $226(1.4 \%)$ & $926(5.6 \%)$ \\
\hline
\end{tabular}




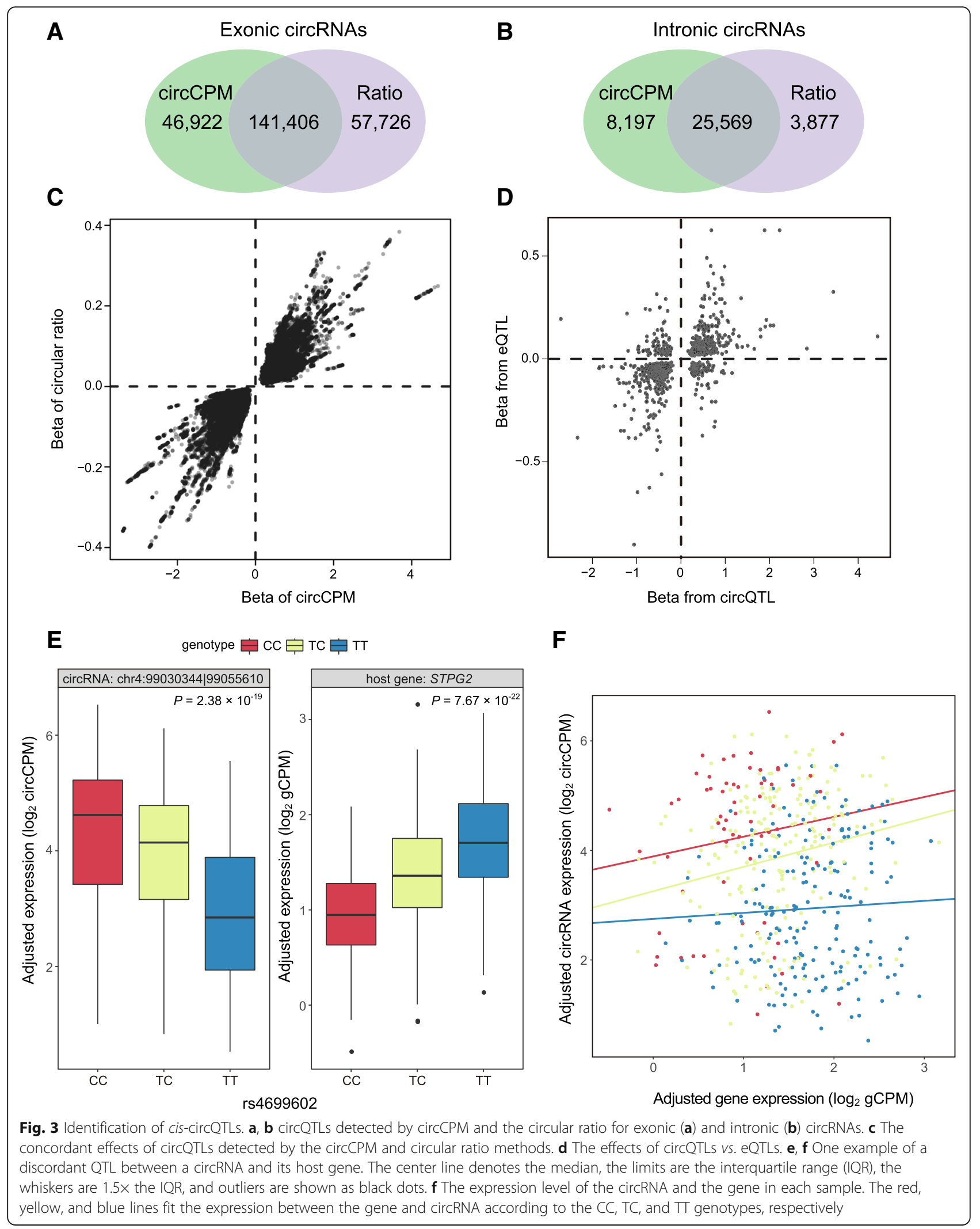


in the same dataset [11], approximately $48.6 \%$ (851 of 1750) of max-circQTLs of circRNAs with an explicit host gene were also eQTLs. For SNPs that were both circQTLs and eQTLs, 77.9\% (663 of 851) exhibited concordant effects (Fig. 3d), while the others were discordant. For the SNPs that are both eQTLs and circQTLs, we observed that there may be an interactive expression relationship between the circRNA and the host gene (Fig. 3e, f).

\section{Potential mechanisms of circQTL}

To study the potential mechanisms of circQTLs, we first analyzed the distance distribution of max-circQTL SNPs to the back-splicing site. We found that the max-circQTLs were preferentially located in proximity to the back- splicing acceptor or donor sites. About 22.1\% (462 of 2086) max-circQTL SNPs were located in flanking intron of junction sites and 20.5\% (427 of 2086) were located in circRNA region. These results suggested that SNPs located in flanking sequences more likely contribute to circRNA regulation (Fig. 4a).

We tested the enrichment of 1721 linkage disequilibrium (LD)-based pruning max-circQTL SNPs against sequence-defined elements by using the two-tailed FET; the MAF and distance-matched non-circQTL SNPs served as controls (see the "Materials and methods" section). We found that intron was the most enriched (Fig. 4b, c; FDR $=4.31 \times 10^{-3}, \mathrm{OR}=3.59$, two-tailed FET) among 11 sequence-defined elements, which suggested

A

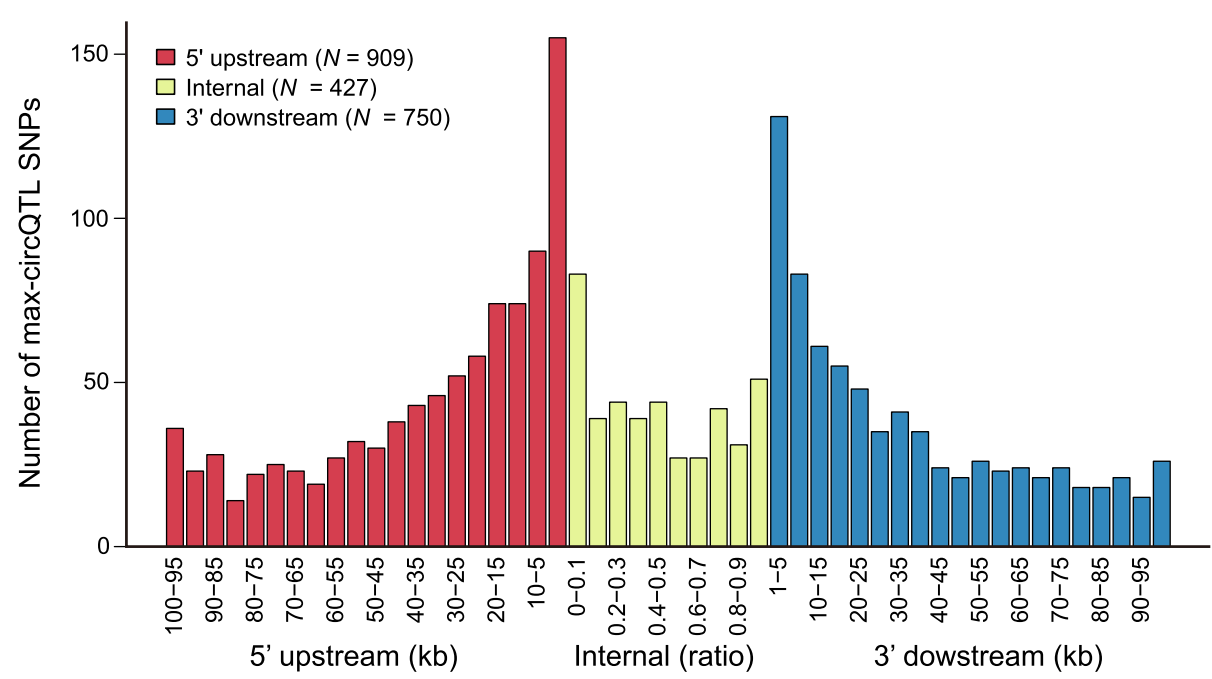

B

circQTL non-circQTL

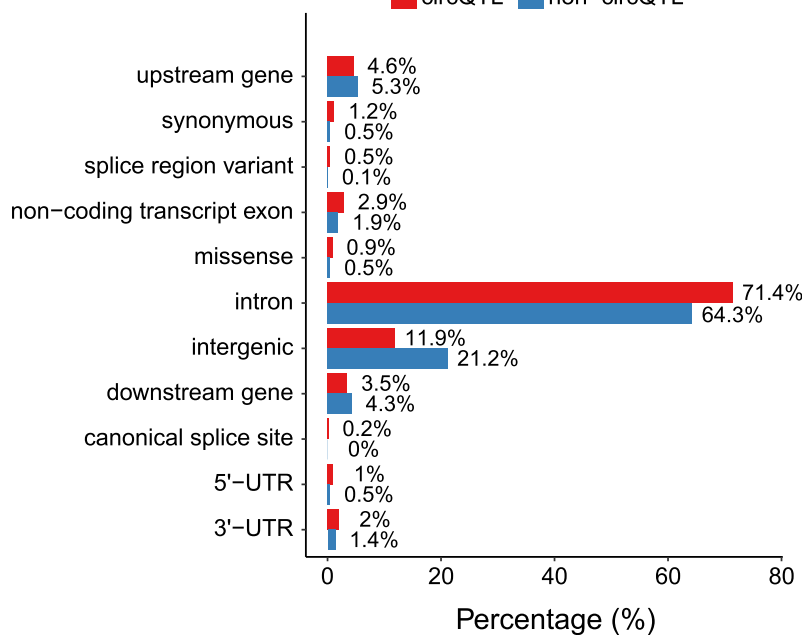

C

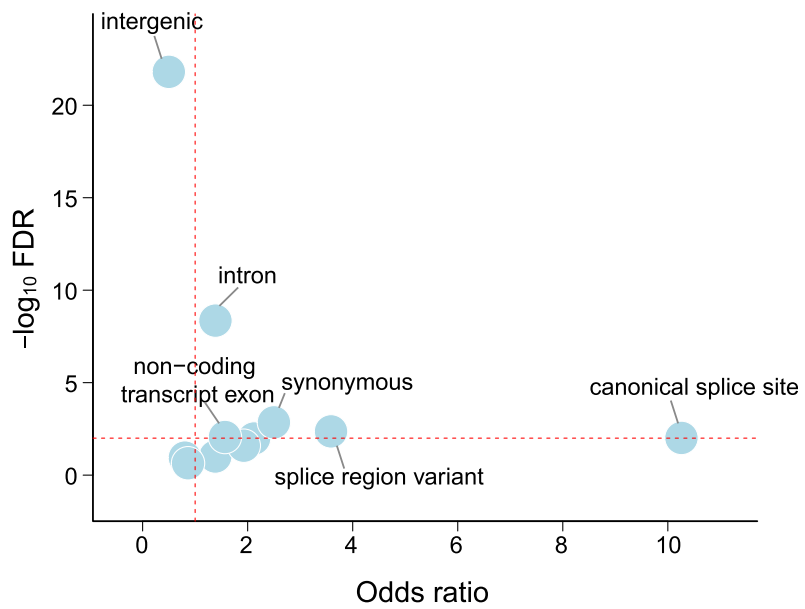

Fig. 4 Distribution of the identified circQTL SNPs. a Distance distribution of the most strongly associated circQTL SNPs for each circRNA (maxcircQTL) to their nearest back-spliced site. For internal max-circQTL SNPS, the distance was calculated by the ratio of the distance between the SNP and the $3^{\prime}$ to $5^{\prime}$ back-splicing site. $\mathbf{b}$ Comparison of the distributions of sequence-defined elements between pruning max-circQTL SNPS and non-circQTL SNPS. c Enrichment of max-circQTL SNPs among sequence-defined elements. The dashed horizontal line indicates an FDR $=0.01$, and the dashed vertical line indicates an odds ratio $(O R)=1$, two-tailed FET 
that introns are implicated in circRNA circularization, as noted in previous studies [32]. A previous study reported that reverse complementary sequences (RCSs) in introns promote circRNA formation [33]. We also found that 1229 pruning max-circQTL SNPs located in introns were enriched in RCSs $\left(11.5 \%, P=1.26 \times 10^{-4}\right.$, $\mathrm{OR}=1.45$, two-tailed FET), with non-circQTL SNPs in introns serving as controls.

Canonical back-splicing signals are required for circularization [18]. Thus, we manually inspected SNPs located in back-splicing sites from all 1,611,445 SNPs for circQTL identification. We detected seven canonical back-splicing SNPs (Additional file 8: Table S7), all of which were identified as circQTL SNPs. The circRNA expression of individuals with the canonical splice genotype was higher than that with the non-canonical splice genotype (Fig. 5a) in all seven SNPs. Four of the seven back-splicing SNPs were also significantly related to their host gene expression with three concordant and one discordant effects [11]. Our results supported that circQTL SNPs located in canonical back-splicing sites may influence circRNA expression by disrupting circRNA formation.

Among the 11 sequence-defined elements, the canonical splice site had the highest OR (Fig. 4c; FDR $=9.73 \times 10^{-3}$, $\mathrm{OR}=10.3$, two-tailed FET). We manually detected 17 SNPs (Additional file 8: Table S7) at the canonical splicing site but not at the back-splicing site that were located in the host genes of regulated circRNAs. All the SNPs effected on the expression and/or splicing of their host genes. For the three circRNA with internal splice site (Fig. 5b), the expression levels were higher in the individuals with canonical splice genotype, implying that internal splicing of circRNA may promote circRNA formation or that circRNAs with retained introns are more likely to be degraded. While for the circRNAs with external splice site (Fig. 5c), the 14 circRNAs were not strictly associated with the canonical splice site, which indicated the complex effects of distant splicing on circRNA formation.

\section{Colocalization between circQTL SNPs and disease risk loci} Thousands of genetic loci harboring disease- and trait-associated variants have been identified by GWAS [34]. However, it is often unclear how the genotype is related to the phenotype. Similar to other QTL analyses, i.e., eQTL [11] and sQTL [26], circQTL may also be helpful for understanding the mechanisms underlying the risk of genetic variants identified by GWAS. Thus, we performed enrichment analysis for pruning max-circQTLs using data from the GWAS Catalog [34], a collection of data from GWAS for various human diseases and traits.

We found that pruning max-circQTL SNPs were significantly enriched among loci associated with diseases compared with non-circQTL SNPs $\left(P=3.92 \times 10^{-17}, \mathrm{OR}=3.53\right.$, one-tailed FET). We further analyzed the enrichment using the data of 21 individual diseases with risk loci $>90$ in the catalog (see "Materials and methods" section) and found significant enrichment of circQTL SNPs among loci associated with 11 diseases (Fig. 5a). Because the CMC dataset derives from the DLPFC of SCZ patients and controls, SCZ-associated loci were the most enriched (Fig. 6a; FDR $=2.00 \times 10^{-5}, \mathrm{OR}=5.28$, one-tailed FET), as expected.

By testing all circQTL SNPs in high linkage disequilibrium $\left(r^{2}>0.8\right)$ with GWAS SNPs (Additional file 9: Table S8), we further identified 157 circRNAs associated with 122 diseases (one circRNA can be involved in multiple diseases). For example, we observed 40, 35, and 21 circRNAs associated with SCZ, inflammatory bowel disease, and type II diabetes mellitus, respectively (Fig. 6b-d). Interestingly, an SCZ-related linear isoform AS3MT ${ }^{\mathrm{d} 2 \mathrm{~d} 3}$ [39] was also regulated by the SCZ risk loci (Fig. 6b), implying a potential mechanism between the linear and circular isoform regulated by SCZ risk loci. Notably, 72.6\% (114 of 157) circRNAs could be regulated by GWAS-linked circQTL SNPs located in flanking introns and circRNA regions, which also suggested the important roles of flanking introns and circRNA regions in pathogenesis. The colocalization between circQTL and GWAS loci provides novel insights into the pathogenesis of complex diseases.

\section{Discussion}

Thousands of genetic variants have been associated with diseases by GWAS. However, how these variants exert their effects on diseases remains largely unknown. QTL analysis is a powerful tool for understanding the mechanisms underlying these genetic variants because the target gene (or DNA functional element) via which a genetic variant leads to disease can be identified by determining whether a risk variant also regulates a transcriptomic quantitative trait, such as the eQTL and sQTL [40, 41]. As a layer of the gene regulatory network, circRNA expression variation may underlie the mechanisms of risk loci. Thus, we introduced circQTLs, which represent the expression levels of circRNAs regulated by genetic variants, to unravel the mechanism of GWAS SNPs. We found that circQTL SNPs were significantly enriched for the GWAS variants associated with various diseases, such as schizophrenia, inflammatory bowel disease, and type II diabetes mellitus. With our systematic analysis of genetically mediated circRNA expression, we show that circQTLs could be used to refine the functional consequences of GWAS loci as another QTL approach. We also tried to identify potential mechanisms of circQTLs and found variants at canonical back-splicing sites in response to lower circRNA expression, implying that canonical splicing signals are required for circularization. In addition to back-splicing 


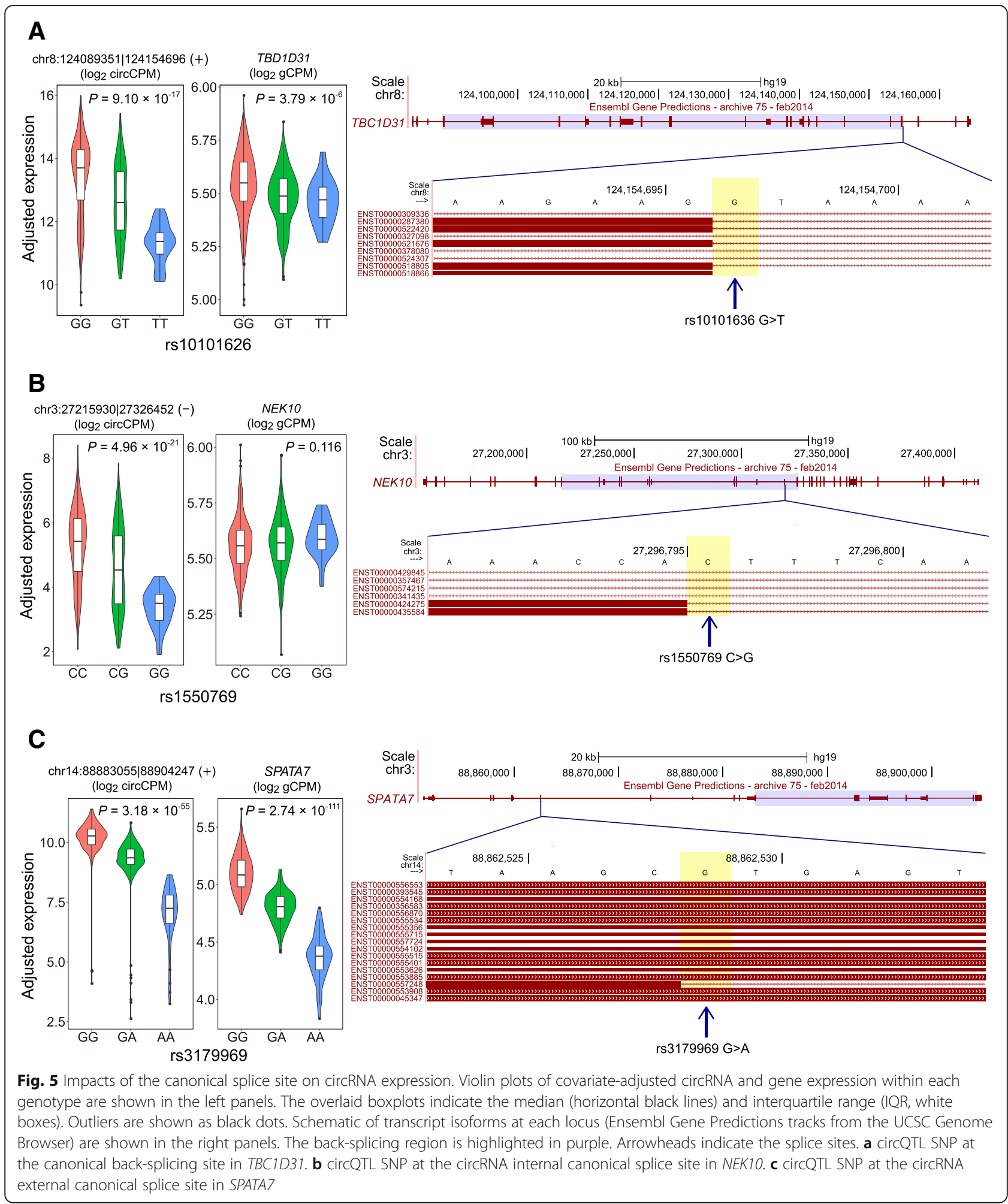

sites, the internal and external splicing sites of a circRNA may contribute to its expression. In addition, we showed that circQTLs were enriched in RCSs, which play important roles in exon circularization [33]. Although we proposed several potential mechanisms, they may explain less than $10 \%$ of circQTLs. Further studies are needed to unravel the mechanisms underlying these circQTLs.

One challenge in analyzing circQTL is to partition circRNA expression from that of its host gene. Here, we 


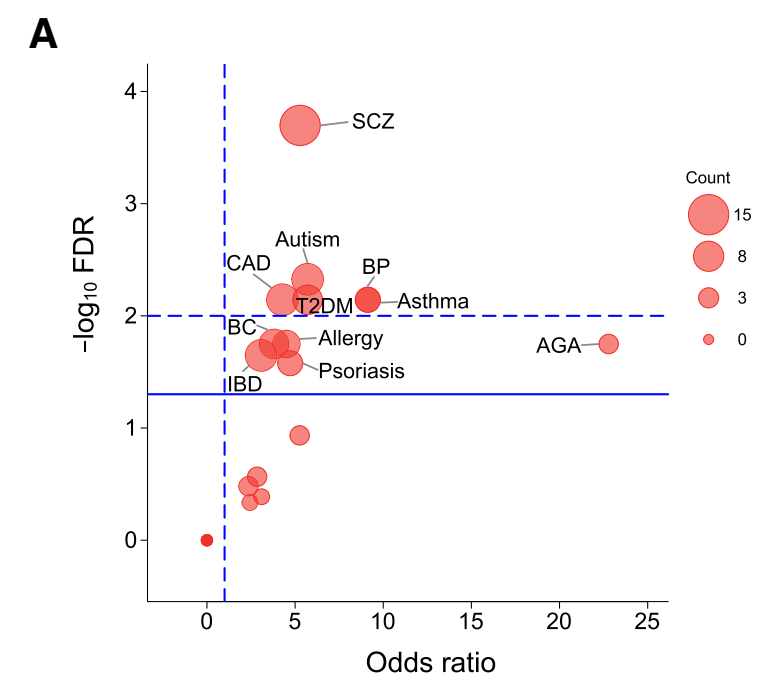

C

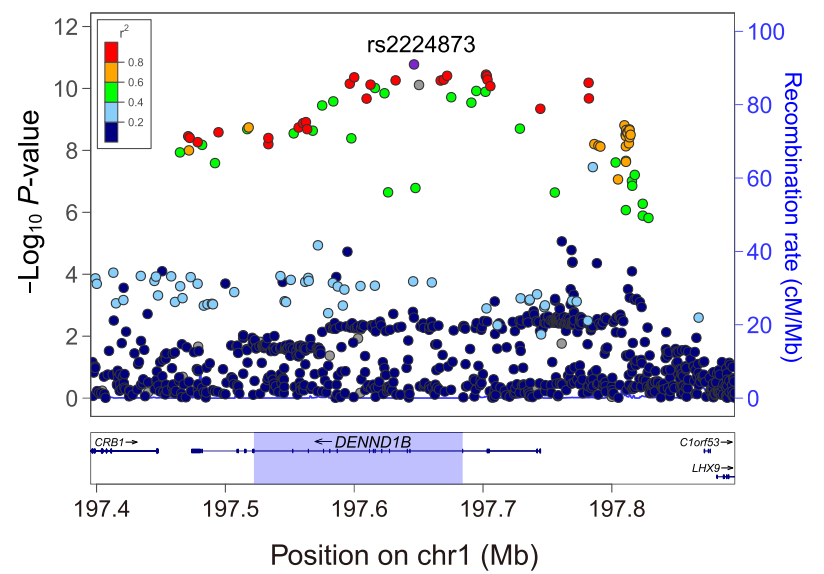

B

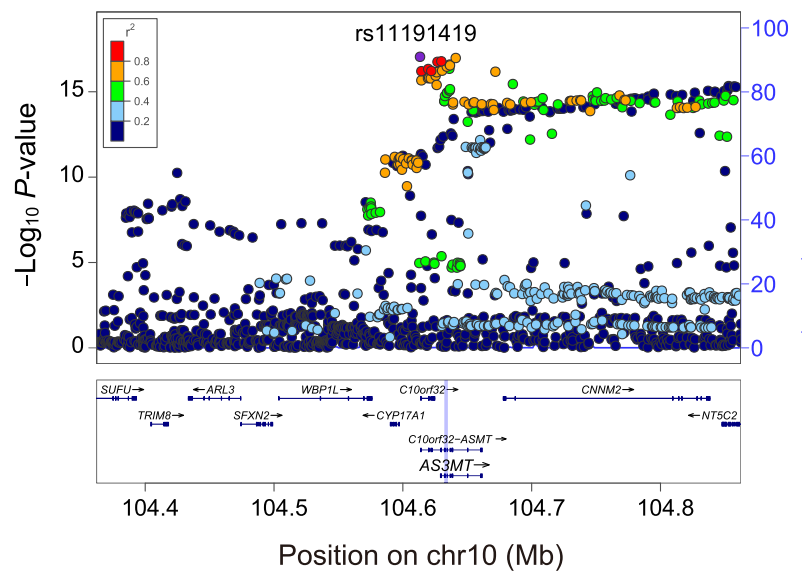

D

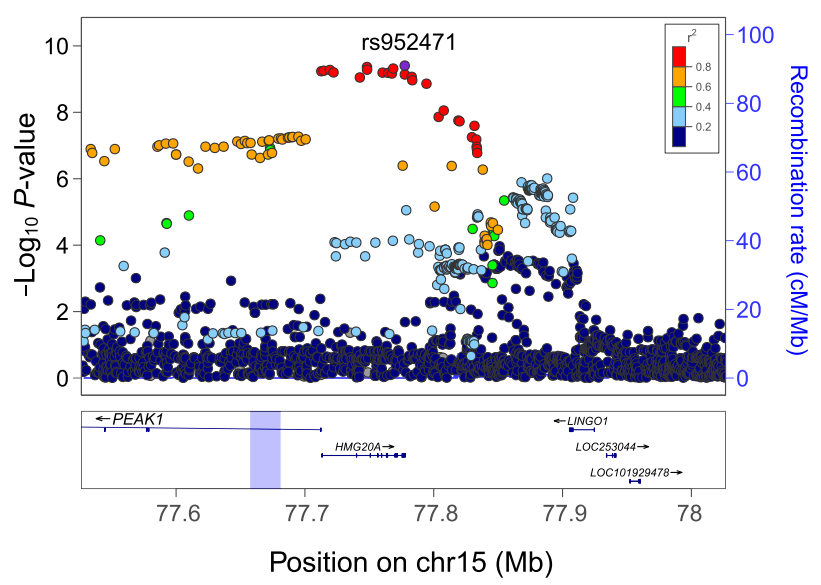

Fig. 6 circQTL SNPs enriched in GWAS loci. a Enrichment of pruning max-circQTL SNPs among the GWAS Catalog. The circle size is proportional to the number of overlapping SNPs between GWAS loci and pruning max-circQTLs. The dashed horizontal line indicates an FDR $=0.01$, the solid horizontal line indicates an FDR $=0.05$, and the dashed vertical line indicates an $\mathrm{OR}=1$, one-tailed FET. AGA: androgenetic alopecia; BC: breast carcinoma; BP: bipolar disorder; CAD: coronary artery disease; IBD: inflammatory bowel disease; T2DM: type II diabetes mellitus. b-d Local plots of circQTL SNPS with high linkage disequilibrium $\left(r^{2}>0.8\right)$ with GWAS results of $\mathbf{b}$ schizophrenia [35], $\mathbf{c}$ inflammatory bowel disease [36], and $\mathbf{d}$ type II diabetes mellitus [37] were generated by LocusZoom [38]. The most significant GWAS SNPs are colored purple. Nearby SNPs are color-coded according to their LD $\left(r^{2}\right)$. The back-splicing region is highlighted in purple. The statistical strength of the association $\left(-\log _{10} P\right.$ values) and recombination rate are double-plotted on the $y$-axis. Genes in the UCSC Genome Browser are shown in the panels below the local plots

identified circQTLs by integrating two methods, the circular junction and circular ratio, to filter out false positives, which are actually eQTLs of the counterpart. Each approach has strengths and weaknesses that complement each other. The advantage of the circular junction approach over the ratio approach is that it detects real circRNA expression levels that play functional roles, while the ratio approach detects only circRNA expression relative to its linear expression. However, the circular junction approach cannot distinguish whether an SNP influences the expression of the host gene or the circRNA itself, while the advantage of the ratio is that the relative value can represent the splicing condition, which directly reflects circRNA formation. Although these two approaches use different quantization strategies, more than $70 \%$ of circQTLs were detected in both approaches with concordant effects.

In addition to genetic factors, i.e., circQTLs, biological and technical factors may also contribute to circRNA expression variation. However, circRNAs are less affected by these factors, mainly because circRNAs are more stable than linear RNAs. The library batch influenced 39\% of circRNAs, indicating that it is a key factor that should be considered in circRNA studies, especially for differential expression analysis. We identified a few circRNAs associated with SCZ (0), PMI (2), and gender (39), but 175 
AOD-related circRNAs were identified, among which 44 circRNAs were shown to be AOD-related independent of their host gene or located in the intergenic region. These circRNAs include CDR1as, which is highly expressed in the human brain [42], chr1:66378928|66384518 from $P D E 4 B$, which interacts with DISC1 to regulate cAMP signaling in schizophrenia [43], and chr2:160193973:160252345 from $B A Z 2 B$, which belongs to the bromodomain gene family associated with transcriptional activation [44]. Considering that circRNAs accumulate in the brain with increasing age [45], these age-related circRNAs may provide new insights into understanding the aging of the human brain or aging diseases.

Dozens of SCZ GWAS risk loci, identified from a large cohort (36,989 cases vs. 113,075 controls) [35], are found to be implicated in circRNA expression, but none of these SCZ GWAS risk circRNAs (circRNA regulated by SCZ GWAS risk loci in circQTL relation) were DE between SCZ and controls in the CMC dataset. Previous study [11] has also encountered this "contradict" in linear gene expression, i.e., no DE evidence was found for SCZ GWAS risk genes (genes regulated by SCZ GWAS risk loci in eQTL relation). For detecting differential expression of SCZ GWAS risk genes at the power of $80 \%$, the median number of subjects with $\mathrm{SCZ}$ and controls was estimated to be 28,500 [11]. Similarly, tens of thousands of samples are also needed for detecting differential expression of SCZ GWAS risk circRNAs, considering the small difference of risk allele frequencies in CMC dataset.

\section{Conclusions}

In summary, our work identified and quantified thousands of circRNAs across hundreds of individuals and focused on the variation of circRNA expression. Unlike linear RNA, circRNA is impacted less by technical and biological effects. Although we detected some circRNAs related to age, mechanistic studies driven by the proposed circRNA candidates will be informative for aging. By performing circQTL analysis, we extended the genetic architecture of disease to circular RNAs. How circRNAs impact the pathogenesis of disease remains to be answered, but our results strongly suggest that circRNAs contribute to disease risk.

\section{Materials and methods Dataset description}

In total, 589 individuals from the CMC database, including 258 SCZ patients, 54 affective/mood disorder patients, and 277 controls, were utilized in this study. Imputation genotyping data (imputed with IMPUTE2 [46]) of autosomes (Synapse: syn3275221) and RNA-seq data (BAM files), including mapped and unmapped reads, were downloaded (Synapse: syn4923029) using the Synapse command line client. Details regarding the RNA-seq analysis and data processing are available at the CMC wiki page (https:// www.synapse.org/\#!Synapse:syn2759792/wiki/69613). The mapped and unmapped reads of each sample were merged and converted into FASTQ format using SAMtools [47] (version 1.3.1).

\section{Detection of circRNA}

The reads in the FASTQ files generated above were mapped onto the reference human genome (hg19) using the BWA [48] memory module with the parameter -T 19. CIRI $[12,13]$ (version 2.0.6) was used to detect putative circRNAs with the parameter $-0-\mathrm{A}$ using the gene annotation file (GENCODE v19, http://www.gencodegenes.org) from each mapped file. CIRI calculates the number of circular junction counts and the circular ratio using the following formula:

$$
\text { Circular ratio }=\frac{C}{C+L}
$$

where $C$ represents the number of circular junction counts of a predicted circRNA and $L$ represents the mean number of reads mapped across the two backsplicing sites but is consistent with linear RNA. circRNAs with circular junction counts $\geq 1$ in more than half of the individuals were considered expressed. In addition, a candidate was eliminated if the average circular ratio $<0.05$, which may be a result of linear splicing byproducts [10].

Besides CIRI, find_circ [4] and CIRCexplorer [15] were also used to identify back-splicing sites. For find_circ, raw reads of RNA-seq were mapped to the reference human genome and unmapped reads were used to detect circular junctions with default parameters [4]. For CIRCexplorer, raw reads of RNA-seq were mapped to the reference human genome by STAR [49] (detailed parameters can be accessed from Additional file 10) and chimeric junction file were parsed with default parameters [15].

\section{Human glioma stem cell culture}

Human glioma stem cell line GSC11 [16] was a gift from Dr. Xing Su and human glioma stem cell G118 was derived from a grade IV glioma on the right temporal lobe in the first affiliated hospital of Soochow University. These cell lines are not listed in the database of commonly misidentified cell lines maintained by ICLAC. GSC11 was authenticated by carrying unique mutations in ATR and ATX genes, and G118 was carrying frameshift in NF1 gene. All the cell cultures performed in this study have been tested as negative for mycoplasma contamination. Both cell lines were cultured under DMEM-F12 medium with N2 and B27. Twenty nanograms/milligram of EGF and bFGF (Sigma) was supplemented to keep the cell 
from differentiation. The culture medium was replaced every other day. All the cell culture-related reagents were from Gibco unless otherwise indicated.

\section{Experimental validation of circRNA}

Thirteen highly expressed circRNAs with annotated adjacent exons (length $>40$ ) of back-splicing were selected for validation (Additional file 4: Table S3). The total RNA of glioma stem cells were extracted by TRIzol (Invitrogen) and reverse transcribed by PrimeScript RT Reagent Kit (Takara). With divergent primers (Additional file 4: Table S3) designed in the two adjacent exons, $50 \mathrm{ng}$ of cDNA template was used for the first round of PCR and 1/50 of the product from the first round of PCR was used as template for the second round of PCR. The final products were analyzed using $2 \%$ agarose gel. The PCR products were further cloned to $\mathrm{pUcm}$-T vector (Sangon) if the bands were clearly visible and the clones with inserts were sequenced by Sanger sequencing (Genewiz).

\section{Evaluate relative circRNA expression level in human cell lines and tissues}

Human Ribo-Zero RNA-seq data of 14 cell lines and 29 tissues were collected from ENCODE Project Consortium [50] (Additional file 1: Table S1). Gene counts were quantified by STAR with annotation file GENCODE v19. circRNAs were detected by CIRI with the same parameters as described in method of detection of circRNA. For each sample, total circular junction counts were calculated by summing circular junction counts of all circRNAs with circular junction counts $\geq 1,2, \ldots, 6$. The relative circRNA expression levels were evaluated by the ratio of total circular junction counts to total gene counts.

\section{Characteristics of the circRNAs}

The identified circRNAs were classified into three types, namely, exonic, intronic, and intergenic, according to their predefined type in CIRI. Notably, a circRNA with one end located in an intergenic or intronic region was categorized as "intergenic" or "intronic" regardless of where the other end was located.

The putative circRNA primary structures were predicted based on human gene annotations (GENCODE v19). Although exons can be removed and introns can be retained during circRNA formation, we considered only exons enclosed by splice sites. The lengths of the circRNAs were calculated by the sum of the exons, and the intron length was added only if it was enclosed by the back-spliced sites.

\section{Normalization and evaluation of covariates}

The voom [20] normalization scales read each circular junction by total read counts across all circular junction sites in the SCZ and control samples and transforms it to the logarithm (base 2). We considered technical (institution, library preparation batch, postmortem interval (PMI), RNA integrity number (RIN), and $\mathrm{RIN}^{2}$ ) and biological (age of death (AOD), ethnicity, gender, SCZ) factors as covariates in voom normalization. We used the limma [21] package (version 3.34.5) to detect DE circRNAs among these covariates. The RIN- and RIN ${ }^{2}$-related circRNAs were merged.

Random sampling analysis was also employed to detect SCZ-associated DE circRNAs. Briefly, 50 or 100 SCZ cases were selected from all cases by stochastic, as well as the equivalent number for controls. SCZ-associated DE circRNAs were detected as described above. The procedure was repeated for 1000 times, and circRNAs reported to be $\mathrm{DE}$ in more than 50 random sampling were identified as SCZ-associated DE circRNAs.

For disease-specific expressed circRNA analysis, circRNAs expressed (circular junction counts $\geq 1$ in more than half of individuals and average ratio $\geq 0.05$ ) in either SCZ or control subjects were selected, and the same method described above was used to detect differentially expressed circRNAs in SCZ.

Raw gene counts were downloaded from the CMC database. We filtered out all genes with low expression in SCZ and control samples, leading to 16,422 genes remaining with at least $1 \mathrm{gCPM}$ (gene counts per million total gene reads) in at least $50 \%$ of the individuals. Then, we used the same method to normalize gene expression and detect covariate-associated genes.

\section{Needed sample size to detect DE circRNAs or genes}

Effect size of each circRNA or gene was estimated by $\mid \log _{2}$ (fold change) $\mid / s d$, whereas fold change is calculated by the ratio of average expression level in SCZ to control, and $s d$ is the standard deviation of all samples expression quantified by $\log _{2}$ (circCPM) for circRNAs or $\log _{2}(\mathrm{gCPM})$ for genes. Cutoff for significance level was estimated by $0.05 / \#$ (circRNAs or genes tested). The needed sample size was estimated by using pwr.t.test function of package pwr in $\mathrm{R}$ with the following parameters: $d=$ max imal effect size, sig.level $=$ cutoff, power $=0.8$, type $=$ "two. sample", and alternative = "two.sided".

\section{circQTL analysis}

We first used voom to normalize all 589 samples using the above method but considered all disease stages as covariates, including SCZ, control, and AFF. Next, 465 genetically inferred Caucasians [11] remained, and the covariate effects were regressed out based on the weight of voom in a weighted linear regression.

The correlations between genotypes and covariate-adjusted circRNA expression, i.e., circRNA expression quantitative loci (circQTLs), were tested using Matrix 
eQTL [28] with the additive linear model on the imputed genotype dosages (only SNPs in autosomal regions were included in this study). SNPs between the 100-kb region upstream of the $5^{\prime}$ back-spliced site and the $100-\mathrm{kb}$ region downstream of the 3' back-spliced site were included for cis-regulation. Similar to a previous study [41], circQTL-containing circRNAs were identified by a permutation procedure that corrects for the multiple hypothesis effect of many variants in LD. Briefly, a total of 10,000 permutations were performed by randomizing sample labels for the circRNA expression matrix. The minimal $P$ value $(\min P)$ for each circRNA in every permutation was collected to derive empirical $P$ values $(\mathrm{emp} P)$ of uncorrected circQTLs for each circRNA. For each uncorrected circQTL $P$ value $\left(P_{\text {circQTL }}\right)$, the emp $P$ can be estimated by the following formula:

$$
\mathrm{emp} P=\frac{1+\sum_{n=1}^{10000} \#\left(\min P_{n}<P_{\text {circQTL }}\right)}{10001}
$$

$\min P_{n}$ represents the $\min P$ in the 10,000 permutations for the circRNA. $Q$ values were calculated using Storey's approach [29] based on the distribution of minimal emp $P$ of all circRNAs. circRNAs with $q$ values less than 0.05 were identified as circQTL-containing circRNAs. For each circQTL-containing circRNA, a emp $P$ equivalent to a $q$ value of 0.05 was deemed the permutation threshold to identify circQTL SNPs.

When measuring the circRNA expression level by the circular ratio, we performed multiple linear regression to regress out covariates and detect correlations between genotypes and the normalized circular ratio.

\section{Analysis of the characteristics of circQTL SNPs}

The most significant SNP for each circQTL-containing circRNA was selected to study the circRNA characteristics. Linked SNPs were pruned with the reference from the 1000 Genomes Phase 1 genotype data [51] using the PLINK [52] -indep-pairwise module with the following parameters: --indep-pairwise 5050.5 . SNPs with minimal uncorrected $P$ values larger than 0.05 were selected as negative controls, i.e., non-circQTL SNPs, after LD-based pruning and MAF distribution correction.

The max-circQTL and non-circQTL SNPs were classified into the following categories: splice acceptor, splice donor, start lost, stop gained, stop lost, missense, splice region, synonymous, untranslated region $5^{\prime}$ (5'-UTR), 3'-UTR, noncoding transcript exon, intron, upstream gene, downstream gene, and intergenic variant, using Variant Effect Predictor (VEP) [53] with the -most_severe parameter. The categories start lost, stop gained, and stop lost were removed due to low counts. In addition, the splice acceptor and splice donor categories were combined into one splicing category, i.e., canonical splice site. Finally, 11 types were retained for enrichment analysis by a two-tailed Fisher's exact test.

\section{Detecting reverse complementary sequences (RCSs)}

We detected RCSs according to a previous study [54]. Briefly, flanking introns of an exonic circRNA were aligned to each other using BLAST [55] (version 2.8.0+) with the parameters -task blastn -word_size 11 -strand minus. Potential RCSs were identified if the bitscores of alignments exceeded 100 .

\section{Datasets of disease-related GWAS SNPs}

The list of SNPs associated with various human traits was downloaded from the GWAS Catalog [34] (http://www.ebi. ac.uk/gwas, the gwas_catalog_v1.0.1 file, accessed Feb 2018). Significant disease-related GWAS SNPs $\left(P \leq 5 \times 10^{-8}\right)$ were extracted using the ontoCAT [56] package of $\mathrm{R}$ if they were associated with the child terms of "EFO_0000408: disease." We performed a one-tailed Fisher's exact test for the following diseases: (1) all human diseases (EFO_0000408: disease); and (2) 21 individual diseases with GWAS SNPs larger than 90, including age-related macular degeneration, allergy, Alzheimer's disease, androgenetic alopecia, asthma, atrial fibrillation, autism, bipolar disorder, breast carcinoma, coronary artery disease, coronary heart disease, inflammatory bowel disease, lung carcinoma, Parkinson's disease, prostate carcinoma, psoriasis, rheumatoid arthritis, schizophrenia, systemic lupus erythematosus, type I diabetes mellitus, and type II diabetes mellitus. SNPs linked $\left(r^{2}>0.6\right.$ calculated by PLINK) to these GWAS SNPs were also included in the enrichment analysis.

\section{Additional files}

Additional file 1: Table S1. List of Ribo-zero RNA-seq data from ENCODE Project Consortium used in this study. (XLSX $16 \mathrm{~kb}$ )

Additional file 2: Figure S1. Relative circRNA expression level in 14 cell lines, 29 tissues, and DLPFC. Figure S2. The Sanger sequencing results for circRNAs from AKT3, HOOK3, and MDGA2. Figure S3. Distributions of the exon numbers $(A)$ and lengths (B) of exonic circRNAs. Figure S4. Pie charts indicated the fraction of circRNAs produced from one host gene. Figure S5. LIB-associated circRNAs show higher expression levels and shorter median lengths. Figure S6. Comparison of distributions of effect size of circRNAs (red line) and genes (blue line) in detecting SCZ-control differential expression in CMC dataset. (DOCX $1163 \mathrm{~kb}$ )

Additional file 3: Table S2. List of 10,559 circRNAs. (XLSX 1499 kb)

Additional file 4: Table S3. Details of RT-PCR primers for validation of 13 selected circRNAs. (XLSX $10 \mathrm{~kb}$ )

Additional file 5: Table S4. Results of the gene-set enrichment analysis of host genes. (XLSX $18 \mathrm{~kb}$ )

Additional file 6: Table S5. List of circRNAs associated with age of death at an FDR $<0.05$. (XLSX $30 \mathrm{~kb}$ )

Additional file 7: Table S6. List of 196,255 circQTLs. (XLSX 14566 kb) Additional file 8: Table S7. The detail of circQTL SNPs located in the splicing site. (XLSX 17 kb) 
Additional file 9: Table S8. List of circQTL SNPs linked to GWAS signals in the NHGRI GWAS Catalog. Each index SNP is significantly associated with disease $\left(P \leq 5 \times 10^{-8}\right)$ in GWAS. circQTL SNPs in high linkage disequilibrium $\left(r^{2}>0.8\right)$ with index SNPs were extracted. (XLSX $\left.290 \mathrm{~kb}\right)$

Additional file 10: In-house scripts for circRNA analysis. Scripts of RNAseq, circQTL, and statistical analysis in this study. (ZIP $56 \mathrm{~kb}$ )

\section{Abbreviations}

AFF: Affective/mood disorder; AGA: Androgenetic alopecia; AOD: Age of death; BC: Breast carcinoma; BP: Bipolar disorder; CAD: Coronary artery disease; CDS: Coding sequence; circCPM: Circular junction counts per million circular junction reads; circQTL: Circular RNA quantitative trait loci; circRNA: Circular RNA; CMC: CommonMind Consortium; DE: Differentially expressed; DLPFC: Dorsolateral prefrontal cortex; empP: Empirical $P$ value; eQTL: Expression quantitative trait loci; FDR: False discovery rate; FET: Fisher's exact test; gCPM: Gene counts per million gene reads; GWAS: Genome-wide association studies; IBD: Inflammatory bowel disease; LIB: Library batch; lincRNA: Long intergenic noncoding RNA; MAF: Minor allele frequency; maxcircQTL: The most significant circQTL for each circRNA; minP: Minimal $P$ value; OR: Odds ratio; PMI: Postmortem interval; QTL: Quantitative trait loci; RCS: Reverse complementary sequence; RIN: RNA Integrity number; SCZ: Schizophrenia; SQTL: Splicing quantitative trait loci; T2DM: Type II diabetes mellitus; UTR: Untranslated region

\section{Acknowledgements}

We appreciate the NIMH Repository and Genomics Resources approve our request for data generated by the CommonMind Consortium. The requested data were generated as part of the CommonMind Consortium supported by funding from Takeda Pharmaceuticals Company Limited, F. Hoffman-La Roche Ltd., and NIH grants R01MH085542, R01MH093725, P50MH066392, P50MH080405, R01MH097276, RO1-MH-075916, P50M096891, P50MH084053S1, R37MH057881 and R37MH057881S1, HHSN271201300031C, AG02219, AG05138, and MH06692. Brain tissue for the study was obtained from the following brain bank collections: the Mount Sinai NIH Brain and Tissue Repository, the University of Pennsylvania Alzheimer's Disease Core Center, the University of Pittsburgh NeuroBioBank and Brain and Tissue Repositories, and the NIMH Human Brain Collection Core. CMC Leadership: Pamela Sklar, Joseph Buxbaum (Icahn School of Medicine at Mount Sinai), Bernie Devlin, David Lewis (University of Pittsburgh), Raquel Gur, Chang-Gyu Hahn (University of Pennsylvania), Keisuke Hirai, Hiroyoshi Toyoshiba (Takeda Pharmaceuticals Company Limited), Enrico Domenici, Laurent Essioux (F. Hoffman-La Roche Ltd), Lara Mangravite, Mette Peters (Sage Bionetworks), Thomas Lehner, Barbara Lipska (NIMH).

We appreciate the facility of High-Performance Computing Cluster supported by Dr. Qimin Zhan, Ms. Guyin Dai, and Mr. Shandong Xu, and the computing resources supported by High-Performance Computing Platform of Peking University

\section{Funding}

This study is supported by the National Key R\&D Program of China (2016YFA0503100) to JC, Beijing Municipal Science and Technology Commission (Z181100001518005) to EY, and Peking University Medicine Fund of Fostering Young Scholars' Scientific \& Technological Innovation to EY (BMU2018PY008).

\section{Availability of data and materials}

The RNA-seq data (https://www.synapse.org/\#!Synapse:syn4923029) with corresponding genotype data (https://www.synapse.org/\#!Synapse:syn3275221) that support the findings of this study are available from CommonMind Consortium Knowledge Portal (https://www.synapse.org/\#!Synapse: syn2759792), but restrictions apply to the availability of these data, which were used under license for the current study, and so are not publicly available. Data are however available from the authors upon reasonable request and with permission of CommonMind Consortium, and the CMC accession numbers used in the study are available in the figshare repository (https://doi.org/10.6084/m9.figshare.8026190) [57]. The genotype data of 1000 Genomes Project Phase 1 data analyzed during the current study can be obtained from (ftp://ftp-trace.ncbi.nih.gov/1000genomes/ftp/release/ 20110521) and details of the files used during the current study are available in the figshare repository (https://doi.org/10.6084/m9.figshare.8034134) [58].
Gene annotation file used in the study can be downloaded from (ftp://ftp. ebi.ac.uk/pub/databases/gencode/Gencode_human/release_19/gencode.v19. annotation.gtf.gz). Data used to calculate circQTL related GWAS SNPs were collected from GWAS Catalog (https://www.ebi.ac.uk/gwas/docs/file-downloads, the gwas_catalog_v1.0.1 file, accessed Feb 2018). The accession number of ENCODE RNA-seq data analyzed during the current study can be obtained from Additional file 1. The dataset of detected circRNAs, age of death associated circRNAs, circQTLs, and GWAS circQTL SNPs generated during the current study are included in the Additional files 3, 5, 7, and 9, respectively. The dataset Additional files 1, 2, 3, 4, 5, 6, 7, 8, and 9 are also available in the figshare repository (https://doi.org/10.6084/m9.figshare. 8026229) [59]. The scripts of RNA-seq, circQTL, and statistical analysis are provided in Additional file 10, which are also deposited in (https://github.com/ yangence/circBrain) [60] under a GNU General Public License v3.0.

\section{Authors' contributions}

$\mathrm{ZL}, \mathrm{JC}$, and EY conceived the study and designed the experiments. ZL collected the data from CMC, performed the data analysis with the help from $C T$ and $S L$, and discussed the data with JC and EY. YR and JC designed and conducted experimental validation. ZL, YR, JC, and EY wrote the paper. All authors read and approved the final manuscript.

\section{Ethics approval and consent to participate}

This research was performed in accordance with the World Medical Association Declaration of Helsinki. Ethics approval for the collection and use of glioma stem cells was granted to JC by the Ethics Committee of Soochow University.

\section{Consent for publication}

Not applicable.

\section{Competing interests}

The authors declare that they have no competing interests.

\section{Publisher's Note}

Springer Nature remains neutral with regard to jurisdictional claims in published maps and institutional affiliations.

\section{Author details}

${ }^{1}$ Institute of Systems Biomedicine, Department of Medical Bioinformatics, School of Basic Medical Sciences, Peking University Health Science Center, Beijing 100191, People's Republic of China. ${ }^{2}$ Institute of Functional Nano and Soft Materials (FUNSOM) and Collaborative Innovation Center of Suzhou Nano Science and Technology, Soochow University, Suzhou 215123, People's Republic of China. ${ }^{3}$ Department of Microbiology and Infectious Disease Center, School of Basic Medical Sciences, Peking University Health Science Center, Beijing 100191, People's Republic of China. ${ }^{4}$ Department of Industrial Engineering and Operations Research, School of Engineering and Applied Science, Columbia University, New York, NY 10027, USA. ${ }^{5}$ Key Laboratory of Neuroscience (Peking University), Ministry of Education, Beijing 100191, People's Republic of China.

Received: 5 December 2018 Accepted: 25 April 2019

Published online: 20 May 2019

\section{References}

1. Chen $L-L$. The biogenesis and emerging roles of circular RNAs. Nat Rev Mol Cell Bio. 2016;17:205-11.

2. Glazar P, Papavasileiou P, Rajewsky N. circBase: a database for circular RNAs. RNA. 2014;20:1666-70.

3. Hansen TB, Jensen TI, Clausen BH, Bramsen JB, Finsen B, Damgaard CK, Kjems J. Natural RNA circles function as efficient microRNA sponges. Nature. 2013;495:384-8.

4. Memczak S, Jens M, Elefsinioti A, Torti F, Krueger J, Rybak A, Maier L, Mackowiak SD, Gregersen LH, Munschauer M, et al. Circular RNAs are a large class of animal RNAs with regulatory potency. Nature. 2013;495:333-8.

5. Ashwal-Fluss R, Meyer M, Pamudurti NR, Ivanov A, Bartok O, Hanan M, Evantal N, Memczak S, Rajewsky N, Kadener S. circRNA biogenesis competes with pre-mRNA splicing. Mol Cell. 2014;56:55-66. 
6. Pamudurti NR, Bartok O, Jens M, Ashwal-Fluss R, Stottmeister C, Ruhe L, Hanan M, Wyler E, Perez-Hernandez D, Ramberger E, et al. Translation of CircRNAs. Mol Cell. 2017;66:9-21.

7. Legnini I, Di Timoteo G, Rossi F, Morlando M, Briganti F, Sthandier O, Fatica A, Santini T, Andronache A, Wade M, et al. Circ-ZNF609 is a circular RNA that can be translated and functions in myogenesis. Mol Cell. 2017;66:22-37.

8. Lasda E, Parker R. Circular RNAs: diversity of form and function. RNA. 2014; 20:1829-42.

9. Rybak-Wolf A, Stottmeister C, Glazar P, Jens M, Pino N, Giusti S, Hanan M, Behm M, Bartok O, Ashwal-Fluss R, et al. Circular RNAs in the mammalian brain are highly abundant, conserved, and dynamically expressed. Mol Cell. 2015;58:870-85.

10. Guo JU, Agarwal V, Guo H, Bartel DP. Expanded identification and characterization of mammalian circular RNAs. Genome Biol. 2014;15:409.

11. Fromer M, Roussos P, Sieberts SK, Johnson JS, Kavanagh DH, Perumal TM, Ruderfer DM, Oh EC, Topol A, Shah HR, et al. Gene expression elucidates functional impact of polygenic risk for schizophrenia. Nat Neurosci. 2016;19: 1442-53.

12. Gao Y, Zhang J, Zhao F. Circular RNA identification based on multiple seed matching. Brief Bioinform. 2018;19:803-10.

13. Gao Y, Wang J, Zhao F. CIRl: an efficient and unbiased algorithm for de novo circular RNA identification. Genome Biol. 2015;16:4.

14. Zeng $X$, Lin W, Guo M, Zou Q. A comprehensive overview and evaluation of circular RNA detection tools. PLoS Comput Biol. 2017;13:e1005420

15. Zhang $X O$, Dong R, Zhang Y, Zhang JL, Luo Z, Zhang J, Chen LL, Yang L. Diverse alternative back-splicing and alternative splicing landscape of circular RNAs. Genome Res. 2016;26:1277-87.

16. He H, Nilsson CL, Emmett MR, Marshall AG, Kroes RA, Moskal JR, Ji Y, Colman H, Priebe W, Lang FF, Conrad CA. Glycomic and transcriptomic response of GSC11 glioblastoma stem cells to STAT3 phosphorylation inhibition and serum-induced differentiation. J Proteome Res. 2010;9:2098-108.

17. Huang da W, Sherman BT, Lempicki RA. Systematic and integrative analysis of large gene lists using DAVID bioinformatics resources. Nat Protoc. 2009:4:44-57.

18. Starke S, Jost I, Rossbach O, Schneider T, Schreiner S, Hung L-H, Bindereif A. Exon circularization requires canonical splice signals. Cell Rep. 2015;10:103-11.

19. You X, Vlatkovic I, Babic A, Will T, Epstein I, Tushev G, Akbalik G, Wang M, Glock C, Quedenau C, et al. Neural circular RNAs are derived from synaptic genes and regulated by development and plasticity. Nat Neurosci. 2015;18: 603-10.

20. Law CW, Chen Y, Shi W, Smyth GK. voom: precision weights unlock linear model analysis tools for RNA-seq read counts. Genome Biol. 2014;15:R29.

21. Smyth GK. Linear models and empirical bayes methods for assessing differential expression in microarray experiments. Stat Appl Genet Mol Biol. 2004:3:1.

22. Kefas B, Godlewski J, Comeau L, Li Y, Abounader R, Hawkinson M, Lee J, Fine $\mathrm{H}$, Chiocca EA, Lawler S, Purow B. microRNA-7 inhibits the epidermal growth factor receptor and the Akt pathway and is down-regulated in glioblastoma. Cancer Res. 2008;68:3566-72.

23. Mouradian MM. MicroRNAs in Parkinson's disease. Neurobiol Dis. 2012;46: 279-84

24. Consortium GT, Laboratory DA. Coordinating center - analysis working G, statistical methods groups-analysis working G, enhancing Gg, fund $\mathrm{NIHC}$, Nih/Nci, Nih/Nhgri, Nih/Nimh, Nih/Nida, et al. genetic effects on gene expression across human tissues. Nature. 2017;550:204-13.

25. Huang YC, Hung CF, Lin PY, Lee Y, Wu CC, Hsu ST, Chen CC, Chong MY, Lin $\mathrm{CH}$, Wang LJ. Gender differences in susceptibility to schizophrenia: potential implication of neurosteroids. Psychoneuroendocrino. 2017;84:87-93.

26. Takata A, Matsumoto N, Kato T. Genome-wide identification of splicing QTLs in the human brain and their enrichment among schizophrenia-associated loci. Nat Commun. 2017;8:14519.

27. Raj T, Li YI, Wong G, Humphrey J, Wang M, Ramdhani S, Wang YC, Ng B, Gupta I, Haroutunian V, et al. Integrative transcriptome analyses of the aging brain implicate altered splicing in Alzheimer's disease susceptibility. Nat Genet. 2018;50:1584-92.

28. Shabalin AA. Matrix eQTL: ultra fast $\mathrm{eQTL}$ analysis via large matrix operations. Bioinformatics. 2012;28:1353-8.

29. Storey JD, Tibshirani R. Statistical significance for genomewide studies. Proc Natl Acad Sci U S A. 2003;100:9440-5.

30. Salzman J, Chen RE, Olsen MN, Wang PL, Brown PO. Cell-type specific features of circular RNA expression. PLoS Genet. 2013;9:e1003777.
31. Zhong C, Yu S, Han M, Chen J, Ning K. Heterogeneous circRNA expression profiles and regulatory functions among HEK293T single cells. Sci Rep. 2017;7:14393.

32. Jeck WR, Sorrentino JA, Wang K, Slevin MK, Burd CE, Liu J, Marzluff WF, Sharpless NE. Circular RNAs are abundant, conserved, and associated with ALU repeats. RNA. 2013;19:141-57.

33. Zhang $X O$, Wang HB, Zhang Y, Lu X, Chen LL, Yang L. Complementary sequence-mediated exon circularization. Cell. 2014;159:134-47.

34. Welter D, MacArthur J, Morales J, Burdett T, Hall P, Junkins H, Klemm A, Flicek P, Manolio T, Hindorff L, Parkinson H. The NHGRI GWAS catalog, a curated resource of SNP-trait associations. Nucleic Acids Res. 2014;42:D1001-6.

35. Schizophrenia Working Group of the Psychiatric Genomics C. Biological insights from 108 schizophrenia-associated genetic loci. Nature. 2014;511:421-7.

36. de Lange KM, Moutsianas L, Lee JC, Lamb CA, Luo Y, Kennedy NA, Jostins L, Rice DL, Gutierrez-Achury J, Ji SG, et al. Genome-wide association study implicates immune activation of multiple integrin genes in inflammatory bowel disease. Nat Genet. 2017:49:256-61.

37. Scott RA, Scott LJ, Magi R, Marullo L, Gaulton KJ, Kaakinen M, Pervjakova N, Pers TH, Johnson AD, Eicher JD, et al. An expanded genome-wide association study of type 2 diabetes in Europeans. Diabetes. 2017;66:2888-902.

38. Pruim RJ, Welch RP, Sanna S, Teslovich TM, Chines PS, Gliedt TP, Boehnke M, Abecasis GR, Willer CJ. LocusZoom: regional visualization of genome-wide association scan results. Bioinformatics. 2010;26:2336-7.

39. Li M, Jaffe AE, Straub RE, Tao R, Shin JH, Wang Y, Chen Q, Li C, Jia Y, Ohi K, et al. A human-specific AS3MT isoform and BORCS7 are molecular risk factors in the 10q24.32 schizophrenia-associated locus. Nat Med. 2016;22:649-56.

40. Lappalainen T, Sammeth M, Friedlander MR, 't Hoen PA, Monlong J, Rivas MA, Gonzalez-Porta M, Kurbatova N, Griebel T, Ferreira PG, et al. Transcriptome and genome sequencing uncovers functional variation in humans. Nature. 2013:501:506-11.

41. Ardlie KG, DeLuca DS, Segre AV, Sullivan TJ, Young TR, Gelfand ET, Trowbridge CA, Maller JB, Tukiainen T, Lek M, et al. The genotype-tissue expression (GTEx) pilot analysis: multitissue gene regulation in humans. Science. 2015;348:648-60.

42. Piwecka M, Glazar P, Hernandez-Miranda LR, Memczak S, Wolf SA, RybakWolf A, Filipchyk A, Klironomos F, Cerda Jara CA, Fenske P, et al. Loss of a mammalian circular RNA locus causes miRNA deregulation and affects brain function. Science. 2017:357:1254.

43. Millar JK, Pickard BS, Mackie S, James R, Christie S, Buchanan SR, Malloy MP, Chubb JE, Huston E, Baillie GS, et al. DISC1 and PDE4B are interacting genetic factors in schizophrenia that regulate CAMP signaling. Science. 2005;310:1187-91.

44. Jones MH, Hamana N, Nezu J, Shimane M. A novel family of bromodomain genes. Genomics. 2000;63:40-5.

45. Westholm JO, Miura P, Olson S, Shenker S, Joseph B, Sanfilippo P, Celniker SE, Graveley BR, Lai EC. Genome-wide analysis of drosophila circular RNAs reveals their structural and sequence properties and age-dependent neural accumulation. Cell Rep. 2014;9:1966-80.

46. Howie BN, Donnelly P, Marchini J. A flexible and accurate genotype imputation method for the next generation of genome-wide association studies. PLoS Genet. 2009:5:e1000529.

47. Li H. A statistical framework for SNP calling, mutation discovery, association mapping and population genetical parameter estimation from sequencing data. Bioinformatics. 2011;27:2987-93.

48. Li H, Durbin R. Fast and accurate short read alignment with burrowswheeler transform. Bioinformatics. 2009;25:1754-60.

49. Dobin A, Davis CA, Schlesinger F, Drenkow J, Zaleski C, Jha S, Batut $P$, Chaisson M, Gingeras TR. STAR: ultrafast universal RNA-seq aligner. Bioinformatics. 2013;29:15-21.

50. Consortium EP. An integrated encyclopedia of DNA elements in the human genome. Nature. 2012:489:57-74.

51. Genomes Project C, Abecasis GR, Auton A, Brooks LD, DePristo MA, Durbin RM, Handsaker RE, Kang HM, Marth GT, McVean GA. An integrated map of genetic variation from 1,092 human genomes. Nature. 2012;491:56-65.

52. Purcell $S$, Neale B, Todd-Brown $K$, Thomas L, Ferreira MA, Bender D, Maller J, Sklar P, de Bakker PI, Daly MJ, Sham PC. PLINK: a tool set for whole-genome association and population-based linkage analyses. Am J Hum Genet. 2007; 81:559-75.

53. McLaren W, Pritchard B, Rios D, Chen Y, Flicek P, Cunningham F. Deriving the consequences of genomic variants with the Ensembl API and SNP effect predictor. Bioinformatics. 2010;26:2069-70. 
54. Ivanov A, Memczak S, Wyler E, Torti F, Porath HT, Orejuela MR, Piechotta M, Levanon EY, Landthaler M, Dieterich C, Rajewsky N. Analysis of intron sequences reveals hallmarks of circular RNA biogenesis in animals. Cell Rep. 2015;10:170-7.

55. Altschul SF, Gish W, Miller W, Myers EW, Lipman DJ. Basic local alignment search tool. J Mol Biol. 1990;215:403-10.

56. Kurbatova N, Adamusiak T, Kurnosov P, Swertz MA, Kapushesky M. ontoCAT: an R package for ontology traversal and search. Bioinformatics. 2011;27:2468-70.

57. Liu Z, Ran Y, Tao C, Li S, Chen J, Yang E. Detection of circular RNA expression and related quantitative trait loci in the human dorsolateral prefrontal cortex. 2019. Figshare https://doi.org/10.6084/m9.figshare. 8026190. Accessed 24 Apr 2019

58. Liu Z, Ran Y, Tao C, Li S, Chen J, Yang E. Detection of circular RNA expression and related quantitative trait loci in the human dorsolateral prefrontal cortex. 2019. Figshare https://doi.org/10.6084/m9.figshare. 8034134. Accessed 24 Apr 2019

59. Liu Z, Ran Y, Tao C, Li S, Chen J, Yang E. Detection of circular RNA expression and related quantitative trait loci in the human dorsolateral prefrontal cortex. 2019. Figshare https://doi.org/10.6084/m9.figshare.8026229. Accessed 24 Apr 2019.

60. Liu Z, Ran Y, Tao C, Li S, Chen J, Yang E. Detection of circular RNA expression and related quantitative trait loci in the human dorsolateral prefrontal cortex. 2019. Github Repository. https://github.com/yangence/circBrain. Accessed 24 Apr 2019.

Ready to submit your research? Choose BMC and benefit from:

- fast, convenient online submission

- thorough peer review by experienced researchers in your field

- rapid publication on acceptance

- support for research data, including large and complex data types

- gold Open Access which fosters wider collaboration and increased citations

- maximum visibility for your research: over $100 \mathrm{M}$ website views per year

At BMC, research is always in progress.

Learn more biomedcentral.com/submissions 